\title{
La chimie génératrice de forme : végétation métallique et jardins chimiques
}

\section{Chemistry drawing forms: metallic trees and chemical gardens}

\author{
Florence Haudin, Fabian Brau et Anne De Wit ${ }^{1}$ \\ ${ }^{1}$ Unité de Chimie Physique Non Linéaire, Université libre Bruxelles, boulevard du triomphe, campus de la Plaine, \\ Bruxelles, Belgique. \\ haudin.florence@gmail.com, fabian.brau@ulb.ac.be, adewit@ulb.ac.be
}

\begin{abstract}
RÉSUMÉ. Certaines réactions chimiques produisent un solide et sont capables de générer des structures aux formes étonnantes et caractérisées parfois par des propriétés géométriques remarquables comme l'auto-similarité. Cet article s'intéresse à ces structures, à leur nature esthétique et s'attarde plus longuement sur les jardins chimiques, générés par une réaction de précipitation, revisitée à l'aide d'une géométrie confinée qui produit des tableaux chimiques.

ABSTRACT. Some categories of chemical reactions lead to the formation of solid and are able to produce surprising structures with special geometrical properties such as self-similarity. This paper deals with such structrures, emphasing their aesthetical features and gives more details about chemical gardens, generated by a precipitation reaction, revisited here in a confined geometry producing chemical paintings.

MOTS-CLÉS. Précipitation, végétation métallique, jardins chimiques, confinement.

KEYWORDS. precipitation reaction, metallic tree, chemical gardens, confinment.
\end{abstract}

\section{Création de formes solides et transformations chimiques}

Un état de la matière peut être transformé par un changement de phase thermodynamique. La transformation de l'eau en glace ou le passage d'un métal de la forme liquide à la forme solide sont des exemples familiers. Des réactions chimiques peuvent aussi entraîner des transformations de la matière. Elles peuvent avoir lieu entre deux réactifs en solution ou entre un réactif liquide et un solide. Lors de ces transformations, certaines formes rappelant des végétaux peuvent se former (dendrites dans les roches calcaires, arbres métalliques, jardins chimiques etc...). Cet article est un voyage dans cet univers de la morphogénèse de solides issus de changements de phase par voie chimique. Ces derniers présentent des points communs avec les changements de phase thermodynamiques comme la solidification. Nous introduirons deux types de réactions chimiques susceptibles de créer ou de transformer un solide.

\subsection{La solidification}

Dans certains cas quand une structure solide croît, il y a formation de dendrites (du grec dendron signifiant arbre). Ce type de structure apparait lors de la transition de phase du liquide au solide : la solidification. C'est par exemple le cas dans les premiers stades de la formation de cristaux de glace. Lors du processus de solidification, un front se forme, perpendiculaire à sa direction de propagation et séparant la partie solide de la partie encore liquide. Ce front est instable et sensible aux moindres fluctuations de température ou de concentration chimique. Cela a pour effet d'entraîner des modifications locales de la vitesse du front, générant des ondulations au niveau de l'interface solide-liquide. A partir du sommet d'une de ces ondulations, un cristal principal appelé tronc, peut se former. Généralement des cristaux secondaires apparaissent perpendiculairement au tronc, pour constituer des branches. Celles-ci croissent 
avec une vitesse plus faible. Le processus se répète, créant ainsi une structure auto-similaire, c'est-àdire qu'on retrouve la même structuration à des échelles différentes. Pour illustration, une dendrite de glace est visible à l'oeil nu sur Fig. 1 a tandis que Fig. 1 b est une photographie de fougères nanostructurées de cuivre générées par voie chimique et observées par microscopie électronique à balayage (BAKTHAVATSALAM et al. 2016). La similarité de forme avec une vraie fougère est mise en évidence par comparaison avec Fig.1 c. On peut constater que les différentes dendrites bien qu'observables à des échelles très différentes possèdent la même morphologie en arbres avec ramifications. Comme pour l'exemple des fougères nanostructurées, les dendrites peuvent apparaître dans le cas de changement de phase induit par des processus chimiques comme détaillé dans les paragraphes suivants.
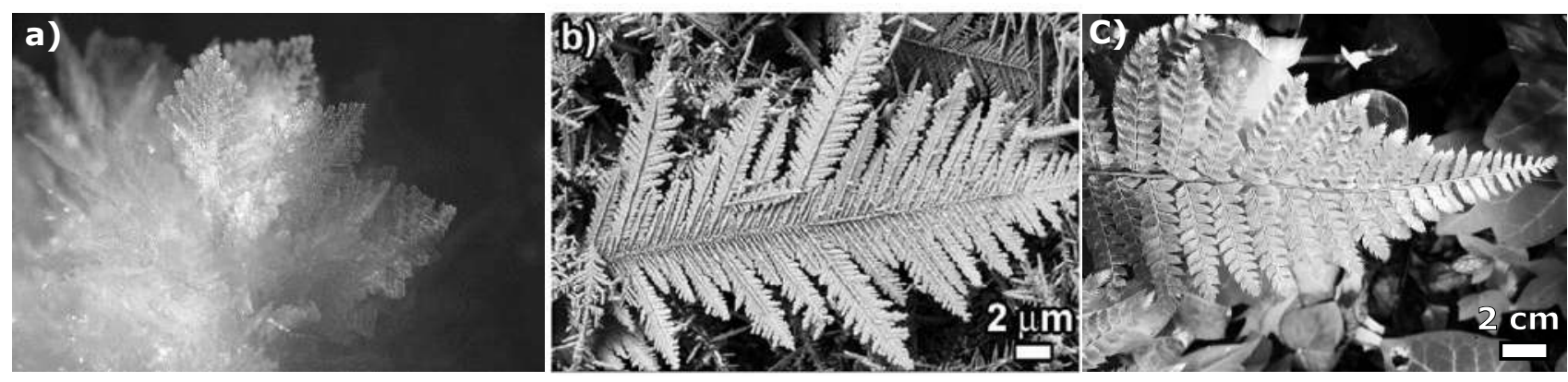

Figure 1.: Exemples de structures dendritiques a) fleurs de glace de taille macroscopique, échelle non précisé, titre : "Fleur de givre", auteur : A. Monnier, fichier source : Fleur_de_givre_L.jpg, Licence CC BY 3.0 desaturée par rapport à l'original), b) dendrites nanostructurées ou fougère de cuivre observées par microscopie électronique à balayage (Source : extrait et modifié à partir de BAKTHAVATSALAM et al. 2016, avec demande d'autorisation à la Royal Society od Chemistry et c) vraie fougère : auteur F. Haudin.

\subsection{Réaction de précipitation}

Une réaction de précipitation est une réaction chimique dont le produit, communément appelé «précipité », est peu soluble dans le solvant contenant les réactifs et génère donc un solide. Ce dernier peut être amorphe (sans ordre à l'échelle microscopique) ou cristallin. Le solide formé peut, selon sa densité par rapport à celle de la phase liquide, soit rester en suspension soit «couler » et venir se déposer au fond du bain. En d'autres termes, la précipitation est l'inverse de la dissolution. Elle peut aussi se produire conjointement à un phénomène de transport de matière comme la convection (mouvements naturels de fluide sous les effets de différences de densité dues à des effets thermiques ou solutaux), l'advection (mouvement forcé du fluide comme dans le cas par exemple de l'injection d'un fluide dans un autre) ou la diffusion (migration lente d'espèce chimique dans un milieu pour homogénéiser la composition chimique du milieu). Ces différents effets peuvent avoir un rôle crucial dans la formation de structures.

\subsection{Réaction d'oxydo-réduction}

L'oxydo-réduction entraîne une transformation de composés par échanges d'électrons. Prenons l'exemple d'un fil de cuivre plongé dans une solution de nitrate d'argent et repris ci-après pour la formation d'arbres métalliques. 
Les demi équations de la réaction et l'équation-bilan sont les suivantes :

$$
\begin{aligned}
A g_{a q}^{+}+e^{-} & \rightarrow A g \\
C u & \rightarrow C u_{a q}^{2+}+2 e^{-} \\
2 A g_{a q}^{+}+C u & \rightarrow 2 A g+C u_{a q}^{2+}
\end{aligned}
$$

Dans ce cas, les ions argent jouent le rôle d'oxydant. Ils captent des électrons (réaction d'oxydation) cédés par le métal cuivre. Ce dernier joue le rôle de réducteur (réaction de réduction). Autrement dit, l'argent initialement présent sous forme d'ions en milieu aqueux produit un dépôt métallique. Le cuivre métal est passé quant à lui en solution, où il est sous forme ionique.

\section{Voyage au coeur d'une flore à base de métaux}

\subsection{Arbres métalliques}

Nous venons d'énoncer le principe d'une réaction d'oxydo-réduction. Intéressons-nous à présent aux formes de végétations métalliques, les dendrites, qu'elle peut générer. Dans le cas de la solidification décrit précédemment, c'est la transition de phase qui les génère alors qu'ici c'est une réaction chimique en solution aqueuse. Par exemple, l'arbre de Diane est produit quand on plonge un fil de cuivre dans une solution de nitrate d'argent (Fig. 2 a). Après ajout d'une goutte de mercure, et après quelques heures d'attente, des aiguilles d'argent ou dendrites apparaissent autour du fil et la solution se colore progressivement en bleu : des ions cuivre $\mathrm{Cu}^{2+}$ sont formés et passent en solution. Suivant les métaux impliqués, les arbres ont des noms différents par référence à l'alchimie qui associe aux métaux du groupe I des divinités de la mythologie grecque (Arbre de Mars : métal fer (limaille) + ajout de liqueur alcaline de tartre (catalyseur); Arbre de Saturne : métal zinc + solution d'acétate de plomb ; Arbre de Jupiter : métal zinc + solution d'ions étain).
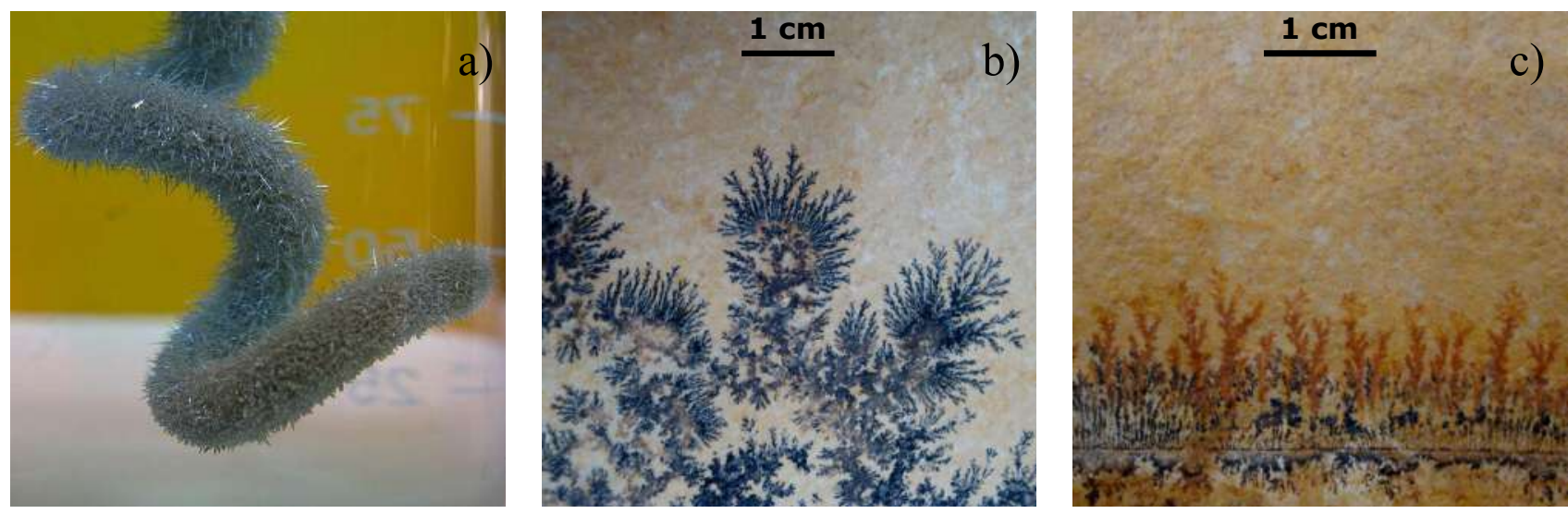

Figure 2.: Un exemple d'arbre et de dendrites métalliques : a) arbre de Diane formé en plongeant un fil de cuivre dans une solution de nitrate d'argent. Titre : "Arbre de Diane (réaction avancée)", auteur : $\Lambda \Phi \Pi$, fichier source : Arbre de Diane (réaction avancée) 19.jpg, Licence CC BY 4.0, échelle non précisée, b) Dendrites de manganèse dans une roche calcaire et c) dendrites de fer et de manganèse ayant grandi perpendiculairement à une fracture : auteur : F. Haudin. 


\subsection{Dans les roches calcaires}

Des structures dendritiques croissent dans certaines roches sédimentaires calcaires : il s'agit d'arborescences de micro-cristaux de manganèse ou de fer. Nous pouvons en voir deux exemples sur les Fig. $2 \mathrm{~b}$ et $\mathrm{c}$. Elles ressemblent à de petits végétaux. Elles apparaissent lorsque l'eau riche en manganèse et en fer, oxydés par la présence d'oxygène dissout et de bactéries, circule dans les fractures de la roche et les interstrates. Plus précisément, les dendrites se forment par dépôt dans les pores de la roche, larges de quelques micromètres : les pores constituent donc un système ramifié dans la roche, avec comme ciment des matériaux à base de manganèse et de fer. Le phénomène responsable de ces dépôts serait la précipitation. Une hypothèse sur son origine serait une augmentation de $\mathrm{pH}$ comme une conséquence de la dissolution du carbonate de calcium (solution basique donc de $\mathrm{pH}$ supérieur à 7). Ces précipitations se produisent à partir de dépôts plus anciens et de même composition ou à partir de nouveaux points de nucléation, avec pour phénomène de transport clé la diffusion : en effet, les composants diffusent radialement à partir des points de nucléation d'origine. De cette façon, des dendrites en forme d'arbres ou d'étoiles sont produites. Une fois formée, un nouvel apport en manganèse va conduire à une épaississement et à un élargissement des tiges et des branches. Le même processus permet d'expliquer la formation des dendrites de fer mais leur croissance à des stades plus avancés est plus difficile à atteindre.

\subsection{Jardins chimiques}

D'autres analogies de forme entre des structures chimiques et des végétaux existent. En dehors des structures dendritiques rappelant des arbres, le cas des jardins chimiques est fascinant. Il s'agit de structures en forme de filaments, rappelant les herbes frêles d'un jardin. Elles sont produites par une réaction chimique de type précipitation, c'est-à-dire capable de produire un solide. Une illustration en est donnée sur la Fig. 3 a, réalisée dans notre laboratoire, avec un bécher rempli de silicate de sodium et quelques grains de sel métallique, du chlorure de cobalt essentiellement.

\section{La version traditionnelle}

\section{Croissances chimiques}

Les structures de type jardins chimiques ont été obtenues et observées pour la première fois au 17ème siècle par Johann Glauber (GLAUBER 1646). Leur croissance spontanée et leur esthétisme ont depuis suscité beaucoup de curiosité. Elles font régulièrement l'objet d'exposition ou sont le sujet de photos artistiques (LES ATOMES CROCHUS). Trois phénomènes agissant de concert sont responsables de leur formation : la réaction de précipitation, la poussée d'Archimède et la pression osmotique (induite par la différence de concentration chimique de part et d'autre d'une membrane semi-perméable). Au début du 20 ème siècle, les jardins chimiques ont été étudiés par un biologiste et chimiste français Stéphane Leduc qui pensait, par leur intermédiaire, avoir identifié les mécanismes chimiques du vivant. Dans ses ouvrages, « les mécanismes de la vie » ou «la biologie synthétique », il décrit notamment les nombreuses croissances osmotiques qu'il a étudiées (LEDUC 1911). Les progrès de la biologie dans le milieu des années 50, mettra un terme définitif à cette vision et à la possibilité de «créer de la vie » à partir de composés inorganiques. 


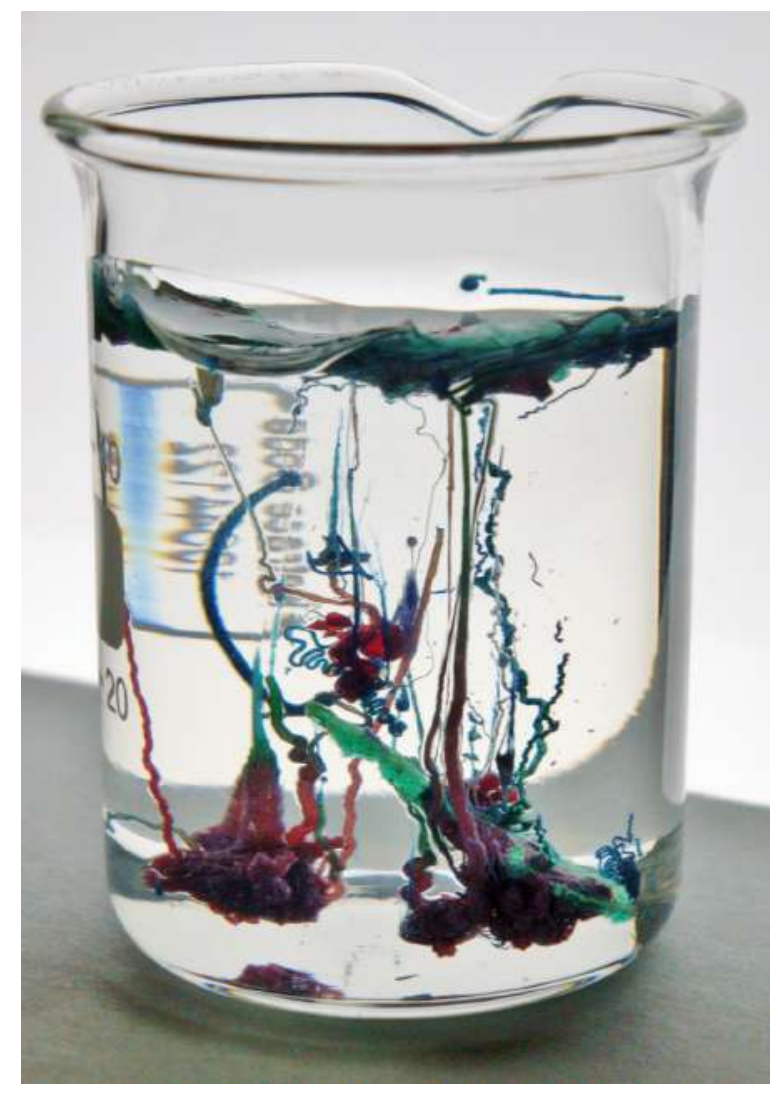

Figure 3.: Exemple de jardin chimique : tiges ayant grandi de façon erratique à partir graines de sel métallique semées dans un bécher rempli de silicate de sodium.

\section{Mécanismes de formation}

Dans sa version traditionnelle, l'expérience consiste à «semer une graine » de sel métallique dans un verre liquide (silicate de sodium) ou une autre solution basique (à base de carbonate, d'oxalate...), véritable terreau alcalin qui initie la croissance de structures tubulaires. En plaçant le sel métallique dans le bain aqueux alcalin, celui-ci se dissout et forme une coque de précipité autour de la graine. Cette coque est semi-permable et peut laisser passer l'eau de la solution extérieure qui est «pompée » sous l'effet de la pression osmotique. L'apport d'eau permet à la dissolution du sel métallique de se poursuivre augmentant la pression à l'intérieur de la coque jusqu'à ce que celle-ci rompe. La solution contenue à l'intérieur de la coque, généralement moins dense, est expulsée vers le haut sous forme de jet. Au contact de la solution alcaline, la partie extérieure de ce jet précipite pour former des structures tubulaires de forme plus ou moins régulière.

\section{Les variantes}

Depuis la version traditionnelle, l'expérience originale a connu des adaptations à des fins de recherches (BARGE L. et al. 2015). Un des buts visés est, par exemple, obtenir une croissance contrôlée des structures tubulaires, en remplaçant notamment le solide de sel métallique par une solution aqueuse et en l'injectant. Cette procédure permet de contrôler non seulement les concentrations mais aussi le débit avec lequel la solution est injectée (THOUVENEL-RomAns et STEINBOCK 2003). Cependant, ces structures restaient tubulaires et tridimensionnelles. Nous avons mis en place une expérience similaire, à base des réactifs habituels utilisés pour les jardins chimiques mais dans laquelle, nous avons réalisé des in- 
jections en milieu confiné : ceci réduit la dimensionnalité des écoulements et des structures produites. Notre objectif était d'aller plus loin dans le contrôle de la formation de couches de précipités. Dans ces expériences, il est possible de capitaliser sur le nombre de paramètres accessibles que l'on peut faire varier (concentrations, vitesse d'injection, confinement). Une autre option est l'alternance de réactifs différents de façon à former des structures à couches multiples avec la possibilité de produire de nouveaux catalyseurs ou des matériaux aux propriétés anisotropes. Le paragraphe suivant s'attache à décrire avec plus de détails la procédure suivie pour obtenir ces structures.

\section{La version confinée}

Pour réaliser le confinement de l'écoulement, on utilise un dispositif expérimental consistant en deux plaques transparentes en plexiglas entre lesquelles est placé un espaceur permettant de fixer la distance entre les plaques $(0.5 \mathrm{~mm})$. Les plaques ainsi séparées sont maintenues fixes dans un cadre métallique. Pour remplir le dispositif d'un premier réactif, la cellule est d'abord ouverte. On étale alors une nappe d'un premier réactif sur la plaque du dessous. Cette dernière possède un trou auquel est connecté un tube capillaire relié à un pousse-seringue qui permet d'injecter un second réactif. On vient ensuite positionner la plaque supérieure. Une fois celle-ci placée, la cellule est donc remplie du premier réactif et le second peut commencer à être injecté par le tube capillaire. Lors de l'injection, des motifs de précipitation sont générés dans la zone de mélange entre les deux réactifs. Avec du chlorure de cobalt comme sel métallique, différentes couleurs peuvent apparaitre correspondant à différents produits de réaction. Les formes et les couleurs obtenues dépendent des concentrations et de la vitesse d'injection. Notons enfin que la cellule est placée au-dessus d'une table lumineuse (utilisée en dessin ou autre art graphique) permettant un rétro-éclairage. Ainsi, quand la solution initialement contenue dans la cellule est transparente, le fond sera blanc. Les dynamiques obtenues lors de l'injection sont filmées par dessus à l'aide d'une caméra digitale fixée sur un pied. La plupart des photos ont été prises quelques minutes après la fin du processus d'injection avec un appareil réflexe numérique muni d'un objectif macro ou un simple appareil compact.

\section{Des formes chimiques variées}

Une variété de formes a été obtenue en utilisant le dispositif décrit précédemment avec néanmoins certains motifs récurrents. De plus, les transitions semblent progressives entre deux types de motifs lorsque l'on fait varier la concentration du réactif injecté (HAUDIN et DE WIT 2015a). Sur Fig. 4 sont représentées quelques une de ces morphologies : spirales, vers, fleurs, filaments, étoiles... La première ligne contient le cas noté «injection directe» pour lequel du chlorure de cobalt (en rose) est injecté dans du silicate de sodium (solution transparente). La seconde ligne contient le cas «injection inversée », obtenu en permutant le réactif injecté et celui déplacé. Notons qu'en général, les motifs sont différents dans le cas direct et inversé (HAUDIN et al. 2015b) A. On le voit par exemple très clairement sur Fig. 4 d et $\mathrm{h}$ où la réaction chimique est associée à une instabilité (réactif moins visqueux déplaçant un fluide plus visqueux). La précipitation a alors lieu à l'intérieur de digitations ce qui n'est pas le cas dans la version inversée de l'expérience pour laquelle l'écoulement est stable (HAUDIN et DE WIT 2015a). 

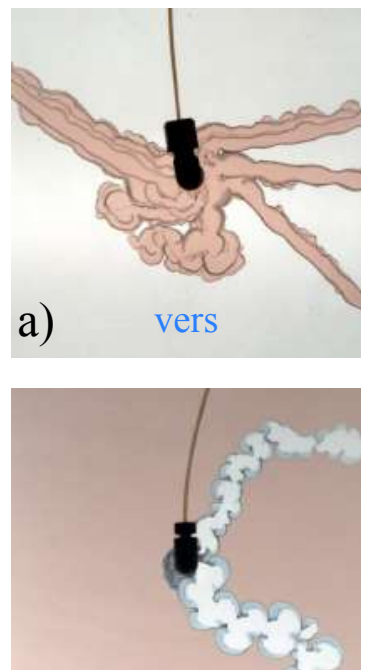

e) spirales
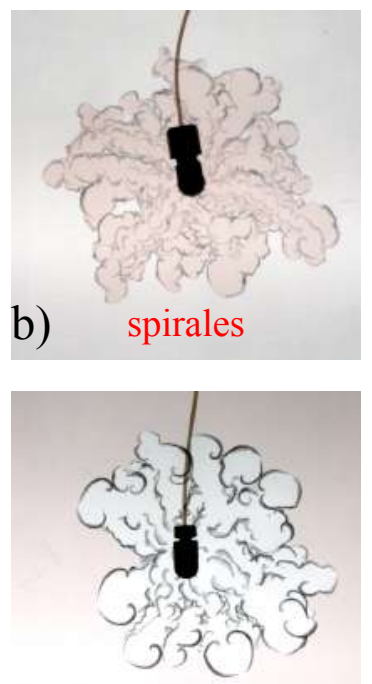

f)

spirales

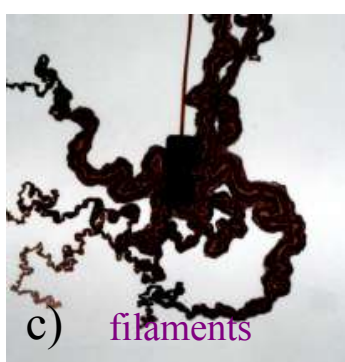

d)
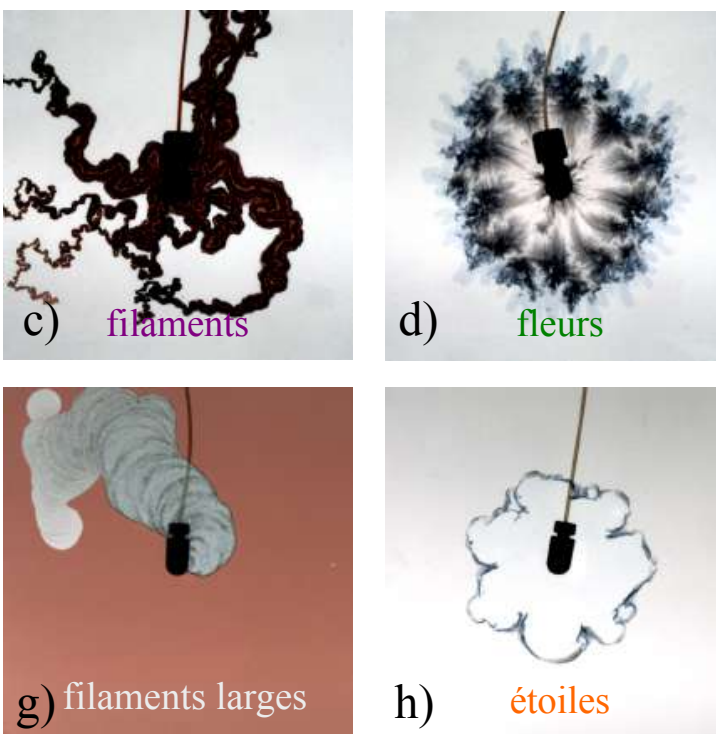

Figure 4.: Exemples de morphologies de précipités obtenus dans une expérience de jardins chimiques en milieu confiné pour le cas d'une injection directe (ligne1) et inversée (ligne 2) a)-e) $C_{C o}=0.63 \mathrm{M}$ et $C_{S i}=0.63 \mathrm{M}$; b)-f) $C_{C o}=0.25 \mathrm{M}$ et $C_{S i}=0.63 \mathrm{M} ; \mathrm{c}$ )-g) $C_{C o}=1 \mathrm{M}$ et $C_{S i}=3.13 \mathrm{M}$ et d)-h) $C_{C o}=0.1 \mathrm{M}$ et $C_{S i}=6.25 \mathrm{M}$. Le débit est le même pour toutes les expériences et égal à $0.11 \mathrm{~mL} / \mathrm{s}$. Le champ de vue des différentes images est de $15 \mathrm{~cm}$ par $15 \mathrm{~cm}$.

\subsection{Des spirales}

Pour toute une gamme de concentrations, les couches de précipité produites ont une forme spiralée comme illustré sur Fig. 4 b et f. Après analyse d'un échantillon de plusieurs dizaines de spirales, il est apparu que ces dernières sont logarithmiques. Ce type de spirales possède un lien avec le nombre d'or et peut être tracé à l'aide de la série de Fibonacci comme illustré sur la construction avec les carrés de la Fig. 5 a. Pour les spirales de l'expérience, leur croissance sous forme de couches de précipité enroulées peut s'expliquer par ajouts successifs de petites quantités de solide à une des extrêmités (HAUDIN et al. 2014). Ce type de croissance logarithmique existe dans d'autres structures naturelles (coquillages, cornes d'animaux etc...). De plus, les spirales logarithmiques sont récurrentes dans la croissance des plantes (les éléments du cœur des fleurs ou les écailles des pommes de pin s'organisent sur des jeux de spirales d'or). On leur associe la notion de proportions harmonieuses, utilisées parfois en art pour la construction de tableaux, pour disposer les différents éléments au mieux (LIVIO 2003).

\subsection{Des paysages abstraits}

En dehors des spirales décrites précédemment, la croissance de jardins chimiques en milieu confiné esquisse d'autres structures aux formes plus ou moins régulières. Le résultat peut s'apparenter à des paysages abstraits. Certains motifs ressemblent à des algues irréelles aux teintes turquoises (Fig. 6 a), à des arbres aux branches piquantes (Fig. 6 b) ou à des tressages de "lianes chimiques" (Fig. 6 c). Sur Fig. $6 \mathrm{~d}$, des structures proches de spirales évoquent des formes aquatiques aux teintes vertes et bleues.

Par ailleurs, certaines structures précipitées sont constituées de couches parallèles entre elles. On peut alors voir se dessiner des effets de relief, avec le même contour solide qui se reproduit, souvent à des échelles différentes, une réplication à l'infini ou presque. C'est par exemple le cas pour des structures en terrasses qui croissent à partir de filaments (Fig. 7 a) ou de vers précipités à un temps antérieur (Fig. 7 b 

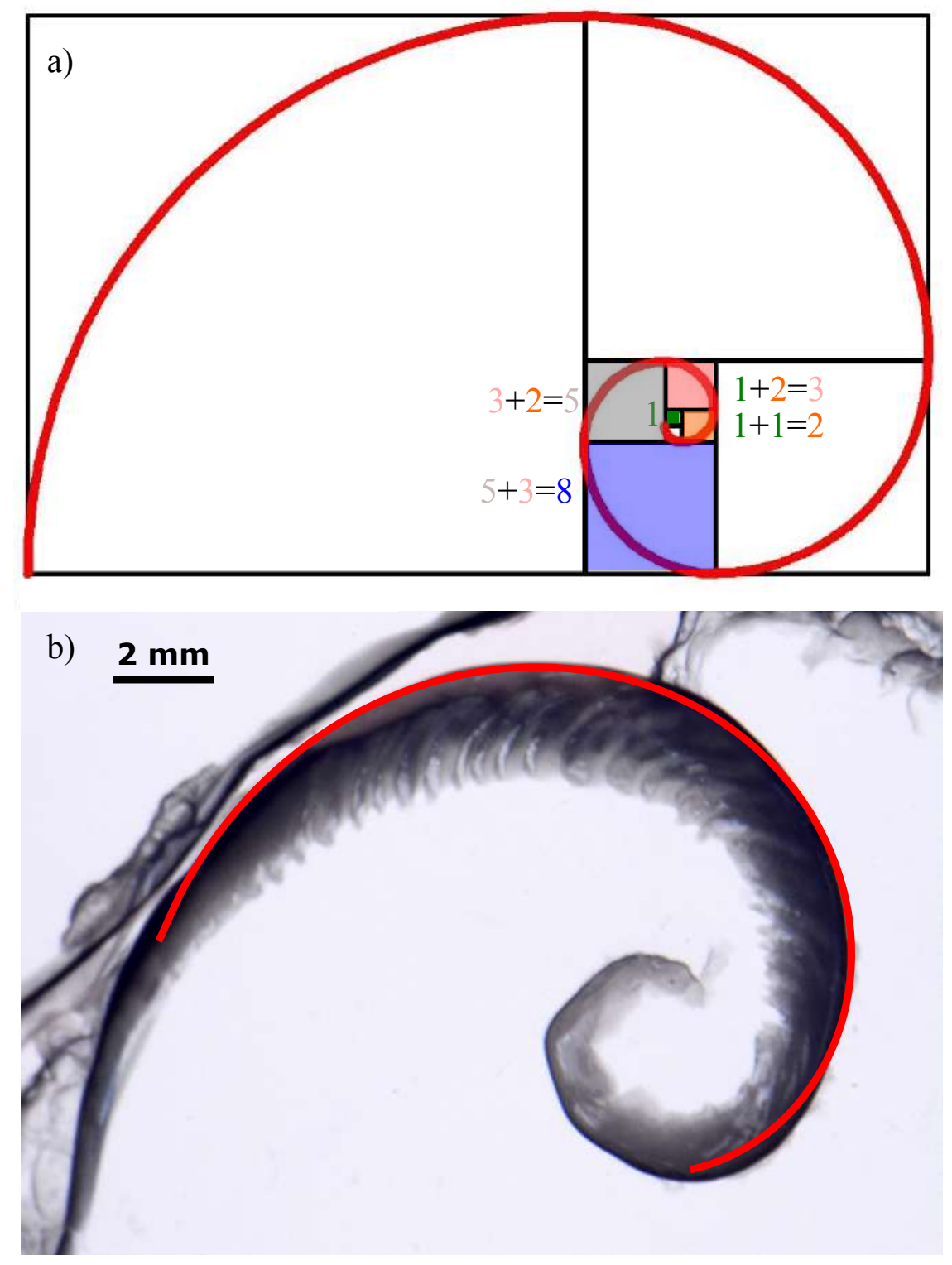

Figure 5.: a) Construction d'une spirale logarithmique avec des carrés contigüs dont les dimensions des côtés décrivent la suite de Fibonacci (les carrés de couleurs réprésentent les 5 premiers termes de la série : 1, 1, 2, 3, 5 et 8) et b) exemple de couche de précipité en forme de spirale logarithmique $\left(C_{S i}=0.63 \mathrm{M}\right.$ et $\left.C_{C o}=0.25 \mathrm{M}\right)$.

et 8 a). Cette multiplication de contours semblables se produit également avec les spirales comme illustré sur Fig. 8 b. Les toiles ainsi esquissées chimiquement évoquent les séries "Conte de pistils et étamines" du peintre abstrait Frantisek Kupka (Fig. 8 c). L'artiste y représente de façon symbolique la fécondation des fleurs dans une vision qui donne le vertige, dans une accumulation de formes et de strates. A la différence du tableau qui présente une plongée vers le centre, les jardins chimiques ont plutôt tendance à donner une illusion de structure en hauteur qui surgit du plan.

Cette impression de relief est aussi présente pour certaines structures de types filaments inversés : ceuxci sont striés de fins contours de précipités, comme si ces grands filaments étaient annelés et articulés à la façon des vers de terre (Fig. 7 c). La Figure 7 d quant à elle, montre des structures qui présentent des plis, produisant un effet de drapé, avec à nouveau la sensation visuelle d'un relief alors que les structures sont confinées dans un espace d'épaisseur un demi millimètre. 

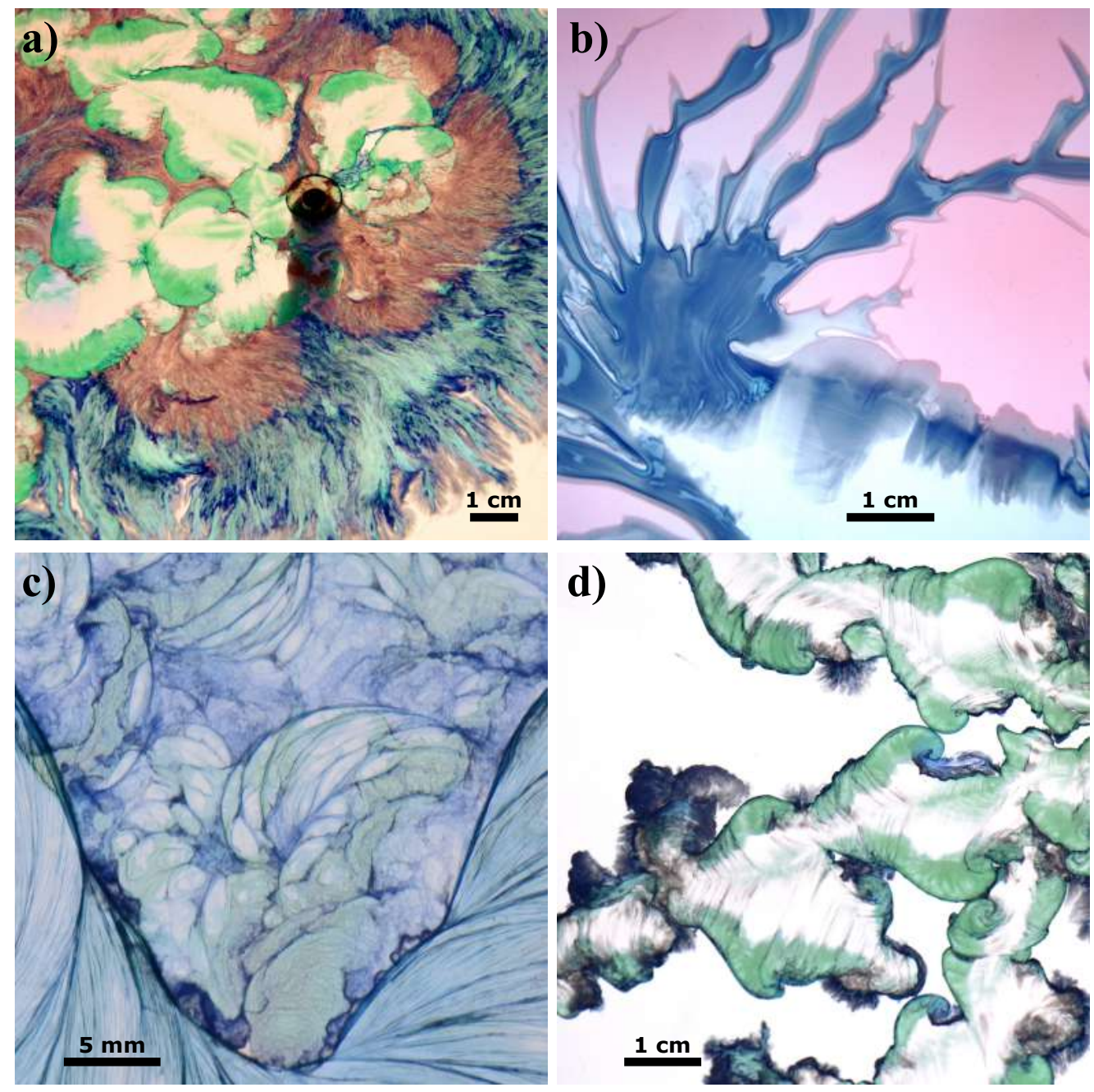

Figure 6.: Paysages abstraits produits par jardins chimiques en milieu confiné obtenus pour les concentrations chimiques suivantes : a) $C_{C o}=0.25 \mathrm{M}$ et $C_{S i}=6.25 \mathrm{M}$, b) $C_{S i}=6.25 \mathrm{M}$ et $C_{C o}=1 \mathrm{M}$, c) $C_{S i}=3.13 \mathrm{M}$ et $C_{C o}=1$ M et d) $C_{C o}=0.2 \mathrm{M}$ et $C_{S i}=6.25 \mathrm{M}$.

\section{Conclusions}

Dans cet article, nous avons montré quelques exemples de structures chimiques formées par réaction chimique créant des solides (dendrites dans les roches, arbres métalliques et jardins chimiques). L'accent a été mis sur les propriétés géométriques de ces structures. Nous avons décrit plus en détails les motifs créés dans l'expérience de jardins chimiques en milieu confiné que nous avons mise au point. Ce procédé permet de générer différents types de motifs quasi 2D en faisant varier les concentrations des deux réactifs. En particulier, des motifs en formes de spirales ou des motifs en couches ou en terrasses sont passés en revue. De façon, un peu plus générale, le confinement permet d'observer des motifs dont certains évoquent des tableaux abstraits. 

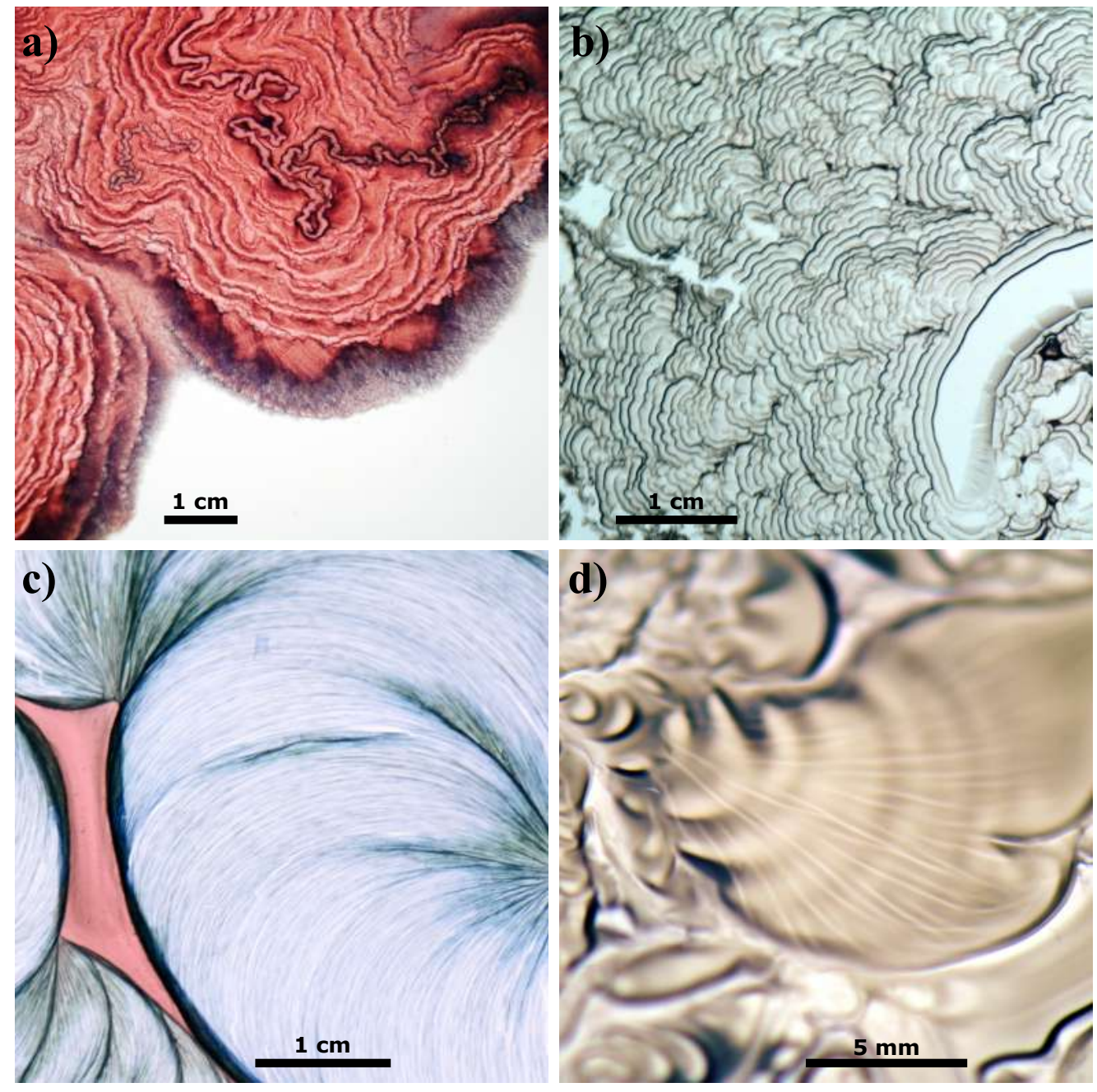

Figure 7.: Effets géométriques : a) structures formées autour de filaments $\left(C_{C o}=0.25 \mathrm{M}\right.$ et $\left.C_{S i}=1.25 \mathrm{M}\right)$ et b) autour de vers pour une solution de sel de calcium colorée en bleu à la place d'un sel de cobalt $\left(C_{C a}=2 \mathrm{M}\right.$ et $\left.C_{S i}=0.63 \mathrm{M}\right)$, c) grand filament strié de lignes avec un effet de coude et de bourrelets $\left(C_{S i}=6.25 \mathrm{M}\right.$ et $\left.C_{C o}=1.38 \mathrm{M}\right)$ et d) impression de plis comme le drapé d'un tissu, avec illusion de relief $\left(C_{S i}=0.63 \mathrm{M}\right.$ et $\left.C_{C o}=0.25 \mathrm{M}\right)$.
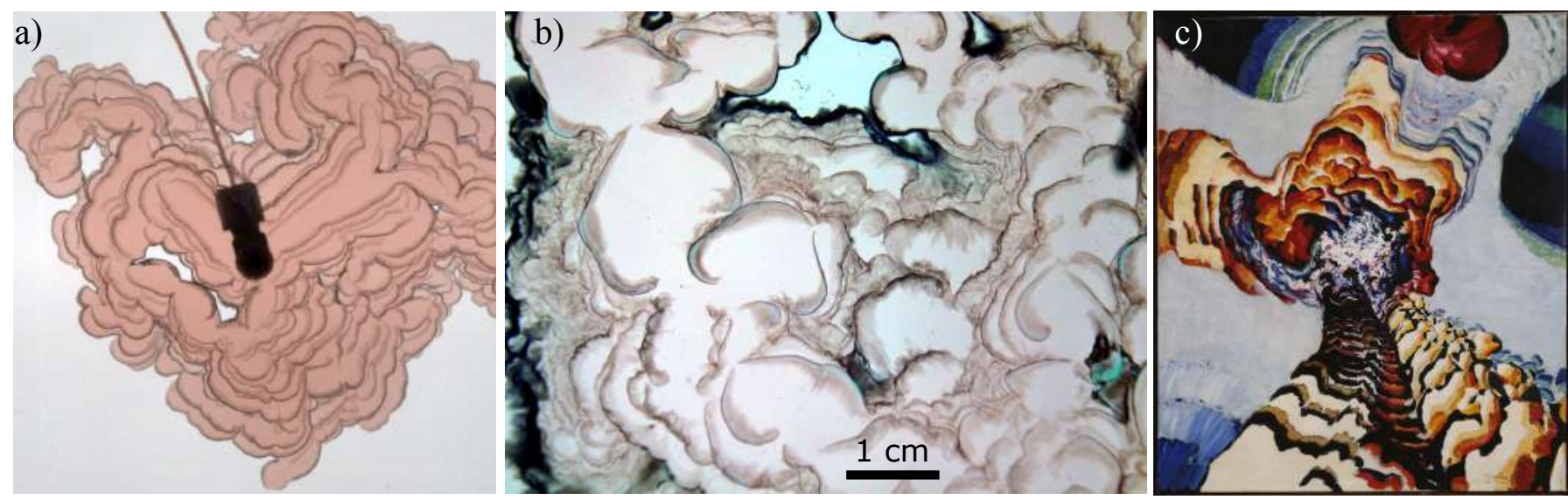

Figure 8.: Une comparaison entre jardins chimiques en milieu confiné et art abstrait : a) motifs en strates autour de structures de types vers $\left(C_{C o}=1 \mathrm{M}\right.$ et $C_{S i}=0.63 \mathrm{M}$, champ de vue : $15 \mathrm{~cm}$ par $\left.15 \mathrm{~cm}\right)$, b) motifs obtenus par des couches de spirales $\left(C_{C o}=0.25 \mathrm{M}\right.$ et $\left.C_{S i}=0.63 \mathrm{M}\right)$ et c), "Conte de pistils et étamines 1 " de F. Kupka ADAGP, Paris, Credit : Photo (C) Centre Pompidou, MNAM-CCI, Dist. RMN-Grand Palais / Jean-Claude Planchet. 


\section{Bibliographie}

BaKthavatsalam R. et al., "Solution Chemistry based Nano-structuring of Copper Dendrites for Efficient use in Catalysis and Superhydrophobic Surfaces", RSC Advances 6, 8416-8430 (2016).

Glauber J.R. , Furni Novi Philosophici (1646), Fabel, Amsterdam.

LES ATOMES CROCHUS https://www.atomes-crochus.org/IMG/pdf/jardins_chimiques.pdf.

LEDUC S., «The mechanism of life » (1911).

BARGE L. et al., "From chemical gardens to chemobrionics.", Chemical Reviews, p.8652, n 115 (2015).

Thouvenel-Romans S. and Steinbock O., "Oscillatory Growth of Silica Tubes in Chemical Gardens", J. Am. Chem. Soc, p.4338-4341, n ${ }^{\circ} 125$ (14) (2003).

HAUdin F. and DE Wit A., "Patterns due to an interplay between viscous and precipitation-driven fingering ", Phys. Fluids p.1113101, n 27 (2015).

Haudin F., Cartwright J.H.E. and De Wit A., "Direct and Reverse Chemical Garden Patterns Grown upon Injection in Confined Geometries", J. Phys. Chem. C. p.15067-15076, n 119 (27) (2015).

HaUdin F., BRAU F., CARTwRIGHT J.H.E. and DE Wit A., "Spirals precipitation patterns in confined chemical gardens", Proc. Nat. Acad. of Sci. p.17363-17367, n 111 (49) (2014).

Livio M., "The Golden Ratio : The Story of $\Phi$, the World's Most Astonishing Number" (2003). 